\title{
GLOBAL EXISTENCE AND CONVERGENCE TO STEADY STATES IN A CHEMOREPULSION SYSTEM
}

\author{
TOMASZ CIEŚLAK \\ Institute of Mathematics, Polish Academy of Sciences \\ Sniadeckich 8, P.O. Box 21, 00-956 Warszawa, Poland \\ E-mail: T.Cieslak@ultra.impan.gov.pl \\ PHILIPPE LAURENÇOT \\ Institut de Mathématiques de Toulouse, CNRS UMR 5219 \\ Université Paul Sabatier (Toulouse III) \\ 118 route de Narbonne, F-31062 Toulouse Cedex 9, France \\ E-mail: laurenco@mip.ups-tlse.fr \\ CRISTIAN MORALES-RODRIGO \\ Institute of Applied Mathematics and Mechanics \\ Faculty of Informatics, Mathematics and Mechanics \\ Warsaw University, Banacha 2, 02-097 Warszawa, Poland \\ E-mail: cristianmatematicas@yahoo.com
}

\begin{abstract}
In this paper we consider a model of chemorepulsion. We prove global existence and uniqueness of smooth classical solutions in space dimension $n=2$. For $n=3,4$ we prove the global existence of weak solutions. The convergence to steady states is shown in all cases.

1. Introduction. In biology some chemicals can induce the movement of living organisms. Such a phenomenon is called chemotaxis. To be more precise, if the organisms move preferably towards regions of high chemical concentration, the motion is called chemoattraction while it is called chemorepulsion if such regions have a repulsive effect on the organisms. Most of the models in the literature are devoted to chemoattraction (cf. $[4,6,7]$ and the survey paper [5]). A salient feature of the chemoattractive case is that finite time blow-up of solutions can take place in space dimension greater or equal

2000 Mathematics Subject Classification: 35K57, 35K60, 92C17.

Key words and phrases: quasilinear reaction-diffusion systems, convergence to equilibrium, compactness method.

The paper is in final form and no version of it will be published elsewhere.
\end{abstract}


to two, and many papers have been devoted to the study of whether and how the finite time blow-up takes place.

This blow-up phenomenon is not expected to take place in chemorepulsion models, such as the following one which is derived in [12] and reads

$$
\begin{cases}u_{t}=\underbrace{\Delta u}_{\text {random motility }}+\underbrace{\nabla \cdot(u \nabla v)}_{\text {chemotaxis }} & \text { in } \Omega \times(0, T), \\ \tau v_{t}=\underbrace{D \Delta v}_{\text {random motility }}-\underbrace{\beta v}_{\text {decay }}+\underbrace{u}_{\text {production }} & \text { in } \Omega \times(0, T), \\ \frac{\partial u}{\partial n}=\frac{\partial v}{\partial n}=0 & \text { on } \partial \Omega \times(0, T), \\ (u, v)(x, 0)=\left(u_{0}, v_{0}\right)(x) & \text { in } \Omega,\end{cases}
$$

where $\Omega$ is an open and bounded subset of $\mathbb{R}^{n}$ with smooth boundary $\partial \Omega, n \geq 2$, and the parameters $\tau, D$ and $\beta$ are positive real numbers.

If $\tau=0$ (that is, there is no term $v_{t}$ in the second equation of $(1)$ ), it is quite easy to see that no finite time blow-up can take place. In fact much more is true and it was proved in $[9,10]$ that solutions exist globally, are uniformly bounded and converge with an exponential rate to the steady state. A similar result would be expected to be valid for (1) but, surprisingly, does not seem to be so easy to prove due to the lack of estimates on $v_{t}$. In particular, global existence of solutions is established in [12] under rather artificial conditions. Indeed, for $n=2$, they require $D$ and $\left\|u_{0}\right\|_{1}$ to fulfil some conditions (cf. [12, A1-A3]). These conditions allow them to construct a Lyapunov functional for (1) in the spirit of that constructed in [4] for the chemoattractive case. For $n \geq 3$ solutions exist globally only under a smallness condition on the initial data in $L^{p}(\Omega)$ with $p>n / 2+1$. To the best of our knowledge, no further result seems to be available for (1).

In the present paper we improve the above-mentioned results in the following directions. First, in space dimension $n=2$ we prove the global existence and uniqueness of uniformly bounded smooth classical solutions without any restriction on the initial data and parameters. In the higher space dimension $n=3,4$, we are only able to establish the global existence of weak solutions. In addition, we prove that there exists a unique steady state up to the mass constraint and it is spatially homogeneous. Our approach relies on the observation that there is a natural Lyapunov functional associated to (1), from which several estimates can be deduced. However, it does not provide any control on $v_{t}$ and does not allow us to obtain smooth classical solutions in space dimension $n \geq 3$.

Notations. The norm in the space $L^{p}(\Omega), 1 \leq p \leq \infty$, is denoted by $\|\cdot\|_{p}$. The classical Sobolev space is denoted by $W^{m, p}(\Omega)$ for $1 \leq p \leq \infty$ and $m \geq 1$ and the associated norm by $\|\cdot\|_{m, p}$. The notation $H^{1}(\Omega)$ is also used for the Hilbert space $W^{1,2}(\Omega)$. If $X$ is a Banach space, $X^{\prime}$ denotes its topological dual space. If $k \geq 1$, the set of $C^{k_{-}}$ smooth functions which vanish on the boundary of $\Omega$ is denoted by $C_{0}^{k}(\Omega)$. Finally, if $T>0, C\left([0, T]\right.$; weak- $\left.L^{1}(\Omega)\right)$ denotes the space of functions from $[0, T]$ in $L^{1}(\Omega)$ which are continuous with respect to time for the weak topology of $L^{1}(\Omega)$. 
We will frequently use the following Gagliardo-Nirenberg inequality

$$
\|w\|_{p} \leq C\|w\|_{1,2}^{\theta}\|w\|_{r}^{1-\theta} \quad \text { with } \quad \theta=\frac{\frac{n}{r}-\frac{n}{p}}{1-\frac{n}{2}+\frac{n}{r}}
$$

which holds true for all $w \in H^{1}(\Omega), p \in[1,2 n /(n-2))$ and $r \in[1, p]$.

Since the existence results to be established in this paper depend strongly on the space dimension, we separate the statements of the results according to the value of $n$.

THEOREM 1.1. Let $n=2$. If $\left(u_{0}, v_{0}\right)$ are non-negative functions in $W^{1, p_{0}}(\Omega)$ for some $p_{0}>2$ then there exists a unique smooth classical bounded uniformly in time solution to (1). Moreover,

$$
\lim _{t \rightarrow+\infty}(u, v)(\cdot, t)=(\bar{u}, \bar{v}) \text { in } \quad C^{2}\left(\bar{\Omega} ; \mathbb{R}^{2}\right) \quad \text { with } \quad \bar{u}=\bar{v}=\frac{1}{|\Omega|} \int_{\Omega} u_{0} d x
$$

The rate of convergence is exponential.

Concerning higher space dimensions, we first introduce the notion of weak solutions to (1) to be used in the sequel.

Definition 1.2. A global weak solution to (1) is a pair of non-negative functions

such that

$$
(u, v) \in C\left([0, \infty) ; \text { weak- } L^{1}\left(\Omega ; \mathbb{R}^{2}\right)\right)
$$

$$
\nabla u, \nabla v, u \nabla v \in L^{1}((0, T) \times \Omega),
$$

and

$$
\begin{aligned}
\int_{\Omega}\left(u(t)-u_{0}\right) \varphi d x+\int_{0}^{t} \int_{\Omega}(\nabla u+u \nabla v) \cdot \nabla \varphi d x d s & =0 \\
\int_{\Omega}\left(v(t)-v_{0}\right) \varphi d x+\int_{0}^{t} \int_{\Omega}(\nabla v \cdot \nabla \varphi+(v-u) \varphi) d x d s & =0
\end{aligned}
$$

for each $t \geq 0$ and $\varphi \in W^{1, \infty}(\Omega)$.

It readily follows from Definition 1.2 that a global weak solution $(u, v)$ to $(1)$ satisfies

$$
\|u(t)\|_{1}=\left\|u_{0}\right\|_{1} \text { and }\|v(t)\|_{1}=e^{-t}\left\|v_{0}\right\|_{1}+\left(1-e^{-t}\right)\left\|u_{0}\right\|_{1} \text { for } t \geq 0 .
$$

We then report the following results:

THEOREM 1.3. Let $n=3$. If $\left(u_{0}, v_{0}\right)$ are non-negative functions in $W^{1, p_{0}}(\Omega)$ for some $p_{0}>3$, then there exists a global weak solution $(u, v)$ to (1) which satisfies also

$$
(u, v) \in L^{5 / 4}\left(0, T ; W^{1,5 / 4}\left(\Omega ; \mathbb{R}^{2}\right)\right)
$$

for any $T>0$. Moreover, recalling that $\bar{u}$ and $\bar{v}$ are defined in Theorem 1.1, we have

for each $\phi \in L^{\infty}(\Omega)$.

$$
\lim _{t \rightarrow+\infty}\left[\int_{\Omega}(u(t)-\bar{u}) \phi d x+\|v(t)-\bar{v}\|_{2}\right]=0
$$

THEOREM 1.4. Let $n=4$. If $\left(u_{0}, v_{0}\right)$ are non-negative functions in $W^{1, p_{0}}(\Omega)$ for some $p_{0}>4$, then there exists a global weak solution $(u, v)$ to (1). Moreover,

$$
\lim _{t \rightarrow+\infty}\left[\int_{\Omega}(u(t)-\bar{u}) \phi d x+\|v(t)-\bar{v}\|_{2}\right]=0
$$

for each $\phi \in L^{\infty}(\Omega)$. 
While the proof of Theorem 1.1 relies on the abstract theory for quasilinear parabolic systems developed in [2], the proof of Theorems 1.3 and 1.4 is performed by a compactness method.

REMARK 1.5. The previous existence results do not seem to extend to space dimension $n \geq 5$ : this is due to the fact that the estimates derived in the next section only allow us to define the product $u \nabla v$ if $n \leq 4$.

As the proofs of our results are the same whatever the values of the positive real numbers $\tau, D$, and $\beta$ are, we set from now on

$$
\tau=D=\beta=1 .
$$

2. Local well-posedness. First, for each $\epsilon \geq 0$, we define the following perturbation of (1):

$$
\begin{cases}u_{t}^{\epsilon}=\Delta u^{\epsilon}+\nabla \cdot\left(u^{\epsilon}\left(1-\epsilon u^{\epsilon}\right) \nabla v^{\epsilon}\right) & \text { in } \Omega \times(0, T), \\ v_{t}^{\epsilon}=\Delta v^{\epsilon}-v^{\epsilon}+u^{\epsilon} & \text { in } \Omega \times(0, T), \\ \frac{\partial u^{\epsilon}}{\partial n}=\frac{\partial v^{\epsilon}}{\partial n}=0 & \text { on } \partial \Omega \times(0, T), \\ \left(u^{\epsilon}, v^{\epsilon}\right)(x, 0)=\left(u_{0}, v_{0}\right)(x) & \text { in } \Omega .\end{cases}
$$

Observe that (1) is obtained by taking $\epsilon=0$ in (4).

THEOREM 2.1. Let $p_{0}>n$ and consider the initial condition $\left(u_{0}, v_{0}\right) \in W^{1, p_{0}}\left(\Omega ; \mathbb{R}^{2}\right)$ with $u_{0}, v_{0} \geq 0$. Then the system (4) has a local unique classical solution

$$
\left(u^{\epsilon}, v^{\epsilon}\right) \in C\left(\bar{\Omega} \times\left[0, t_{\epsilon}^{+}\right) ; \mathbb{R}^{2}\right) \cap C^{\infty}\left(\bar{\Omega} \times\left(0, t_{\epsilon}^{+}\right) ; \mathbb{R}^{2}\right)
$$

and $u^{\epsilon}(x, t), v^{\epsilon}(x, t) \geq 0$ for each $(x, t) \in \bar{\Omega} \times\left[0, t_{\epsilon}^{+}\right), t_{\epsilon}^{+}$denoting the maximal existence time. Moreover, $\left\|u^{\epsilon}(t)\right\|_{1}$ and $\left\|v^{\epsilon}(t)\right\|_{1}$ are given by (3) for $t \in\left[0, t_{\epsilon}^{+}\right)$.

If there is a function $\omega:(0, \infty) \rightarrow(0, \infty)$ such that, for each $T>0$,

$$
\left\|\left(u^{\epsilon}(t), v^{\epsilon}(t)\right)\right\|_{\infty} \leq \omega(T), \quad 0<t<\min \left\{T, t_{\epsilon}^{+}\right\},
$$

then $t_{\epsilon}^{+}=+\infty$. In particular, if $\epsilon \in\left(0, \epsilon_{0}\right]$ with $1 / \epsilon_{0}=\max \left\{\left\|u_{0}\right\|_{\infty},\left\|v_{0}\right\|_{\infty}\right\}$ then $0 \leq$ $u^{\epsilon}, v^{\epsilon} \leq 1 / \epsilon$ and thus $t_{\epsilon}^{+}=+\infty$.

Note that $1 / \epsilon_{0}=\max \left\{\left\|u_{0}\right\|_{\infty},\left\|v_{0}\right\|_{\infty}\right\}$ is finite thanks to the continuous embedding of $W^{1, p_{0}}(\Omega)$ in $L^{\infty}(\Omega)$. Therefore, given $\left(u_{0}, v_{0}\right) \in W^{1, p_{0}}\left(\Omega ; \mathbb{R}^{2}\right)$ with $u_{0}, v_{0} \geq 0$, (4) has a global classical solution for $\epsilon>0$ sufficiently small.

Proof. For $\delta>0$ we define the set $D_{0}:=(-\delta,+\infty) \times(-\delta,+\infty), \mathbf{y}=\left(v^{\epsilon}, u^{\epsilon}\right)$, and $a_{j k} \in C^{\infty}\left(D_{0}, \mathcal{L}\left(\mathbb{R}^{2}\right)\right), 1 \leq j, k \leq n$, by

$$
a=a_{j k}(\mathbf{y})=\left(a_{j k}^{r s}\right)_{1 \leq r, s \leq 2}:=\left(\begin{array}{cc}
1 & 0 \\
u^{\epsilon}\left(1-\epsilon u^{\epsilon}\right) & 1
\end{array}\right) \quad \text { if } j=k,
$$

$a_{j k}(\mathbf{y})=0$ if $j \neq k$. Next for $\mathbf{z} \in D_{0}$ we introduce the operators

$$
\mathcal{A}(\mathbf{y}) \mathbf{z}:=\sum_{j, k=1}^{n}-\partial_{j}\left(a_{j k}(\mathbf{y}) \partial_{k} \mathbf{z}\right), \quad \mathcal{B}(\mathbf{y}) \mathbf{z}:=\sum_{j, k=1}^{n} \nu_{j} \cdot a_{j k}(\mathbf{y}) \partial_{k} \mathbf{z},
$$


and the function $f \in C^{\infty}\left(D_{0} ; \mathbb{R}^{2}\right)$

$$
f(\mathbf{y}):=\left(\begin{array}{c}
u^{\epsilon}-v^{\epsilon} \\
0
\end{array}\right) .
$$

With these notations (4) reads

$$
\begin{aligned}
\partial_{t} \mathbf{y}+\mathcal{A}(\mathbf{y}) \mathbf{y} & =f(\mathbf{y}), \\
\mathcal{B}(\mathbf{y}) \mathbf{y} & =0, \\
\mathbf{y}(0) & =\left(v_{0}, u_{0}\right) .
\end{aligned}
$$

Since $(\mathcal{A}, \mathcal{B})$ is of separated divergence form in the sense of $[2$, Example $4.3(\mathrm{e})]$, then the boundary-value operator $(\mathcal{A}, \mathcal{B})$ is normally elliptic. We can therefore apply $[2$, Theorem 14.4 and Corollary 14.7] to conclude that (4) has a unique maximal classical solution

$$
\mathbf{y}=\left(v^{\epsilon}, u^{\epsilon}\right) \in C\left(\bar{\Omega} \times\left[0, t_{\epsilon}^{+}\right) ; \mathbb{R}^{2}\right) \cap C^{\infty}\left(\bar{\Omega} \times\left(0, t_{\epsilon}^{+}\right) ; \mathbb{R}^{2}\right) .
$$

Moreover, since (with the notations of $\left[2\right.$, Section 15]) $D_{2}=(0,+\infty) \times\{0\}$ and $a_{j j}^{21}=u^{\epsilon}$, $\left(1-\epsilon u^{\epsilon}\right), 1 \leq j \leq n, a_{j k}^{21}=0, a_{j k}^{12}=0$ for $j \neq k, 1 \leq j, k \leq n$ and all these coefficients vanish on $D_{2}$ we can apply [2, Theorem 15.1] to conclude that $u^{\epsilon}(t) \geq 0$ for $\left[0, t_{\epsilon}^{+}\right)$. Next the non-negativity of $v^{\epsilon}$ follows from the standard maximum principle for parabolic equations. The global existence criterion can be deduced from [2, Theorem 15.5]. Finally, if $\epsilon \in\left(0, \epsilon_{0}\right)$, writing the equation solved by $-u^{\epsilon}+1 / \epsilon$, we see that we are in a position to apply [2, Theorem 15.1] to establish that $u^{\epsilon} \leq 1 / \epsilon$. The similar upper bound for $v^{\epsilon}$ is then a straightforward consequence of the classical comparison principle.

We next turn to the existence of a Lyapunov functional for (4) which is the cornerstone of our analysis.

Lemma 2.2. For $\epsilon \in\left[0, \epsilon_{0}\right]$ and $0 \leq s<t<t_{\epsilon}^{+}$the solution $\left(u^{\epsilon}, v^{\epsilon}\right)$ to (4) satisfies the following equality

(5) $F_{\epsilon}\left(u^{\epsilon}(t), v^{\epsilon}(t)\right)-F_{\epsilon}\left(u^{\epsilon}(s), v^{\epsilon}(s)\right)=-\int_{s}^{t} \int_{\Omega}\left(\frac{\left|\nabla u^{\epsilon}\right|^{2}}{u^{\epsilon}\left(1-\epsilon u^{\epsilon}\right)}+\left|\Delta v^{\epsilon}\right|^{2}+\left|\nabla v^{\epsilon}\right|^{2}\right) d x d \tau$ where $F_{\epsilon}$ is given by

if $\epsilon>0$ and

$$
F_{\epsilon}(u, v)=\int_{\Omega}\left(u \ln u+\frac{1}{\epsilon}(1-\epsilon u) \ln (1-\epsilon u)+\frac{|\nabla v|^{2}}{2}\right)
$$

$$
F_{0}(u, v)=\int_{\Omega}\left(u \ln u+\frac{|\nabla v|^{2}}{2}\right) .
$$

Proof. On the one hand, multiplying the first equation of (4) by $\ln u^{\epsilon}-\ln \left(1-\epsilon u^{\epsilon}\right)$ and integrating with respect to space, we obtain

$$
\frac{d}{d t} \int_{\Omega}\left(u^{\epsilon} \ln u^{\epsilon}+\frac{1}{\epsilon}\left(1-\epsilon u^{\epsilon}\right) \ln \left(1-\epsilon u^{\epsilon}\right)\right) d x=-\int_{\Omega} \frac{\left|\nabla u^{\epsilon}\right|^{2}}{u^{\epsilon}\left(1-\epsilon u^{\epsilon}\right)} d x-\int_{\Omega} \nabla u^{\epsilon} \cdot \nabla v^{\epsilon} d x
$$

On the other hand, multiplying the second equation of (4) by $-\Delta v^{\epsilon}$ and integrating with respect to space, we obtain

$$
\frac{1}{2} \frac{d}{d t} \int_{\Omega}\left|\nabla v^{\epsilon}\right|^{2} d x=-\int_{\Omega}\left|\Delta v^{\epsilon}\right|^{2} d x-\int_{\Omega}\left|\nabla v^{\epsilon}\right|^{2} d x+\int_{\Omega} \nabla u^{\epsilon} \cdot \nabla v^{\epsilon} d x .
$$

The expected result then follows by adding (6), (7) and integrating in time. 
As a consequence of Lemma 2.2 we have the following useful inequality.

Corollary 2.3. For $\epsilon \in\left[0, \epsilon_{0}\right]$ and $t \in\left[0, t_{\epsilon}^{+}\right)$, the solution $\left(u^{\epsilon}, v^{\epsilon}\right)$ to (4) satisfies

$$
\int_{\Omega}\left(u^{\epsilon}(t)\left|\ln u^{\epsilon}(t)\right|+\frac{\left|\nabla v^{\epsilon}(t)\right|^{2}}{2}\right) d x+\int_{0}^{t} \int_{\Omega}\left(\frac{\left|\nabla u^{\epsilon}\right|^{2}}{u^{\epsilon}}+\left|\Delta v^{\epsilon}\right|^{2}+\left|\nabla v^{\epsilon}\right|^{2}\right) d x d s \leq C_{0},
$$

where $C_{0}$ depends only on $\Omega$ and $F_{0}\left(u_{0}, v_{0}\right)$.

Proof. On the one hand, since

$$
\begin{aligned}
& r+\frac{1}{\epsilon}(1-\epsilon r) \ln (1-\epsilon r) \geq 0 \quad \text { for } r \in\left[0, \frac{1}{\epsilon}\right], \\
& 2 r \ln r \geq-\frac{2}{e} \quad \text { for } r \in[0,1],
\end{aligned}
$$

we infer from (3) that

$$
\begin{aligned}
F_{\epsilon}\left(u^{\epsilon}(t), v^{\epsilon}(t)\right) & \geq \int_{\Omega}\left(u^{\epsilon}(t) \ln u^{\epsilon}(t)-u^{\epsilon}(t)+\frac{\left|\nabla v^{\epsilon}(t)\right|^{2}}{2}\right) d x \\
& \geq \int_{\Omega}\left(u^{\epsilon}(t)\left|\ln u^{\epsilon}(t)\right|+\frac{\left|\nabla v^{\epsilon}(t)\right|^{2}}{2}\right) d x-\left(\left\|u_{0}\right\|_{1}+\frac{2|\Omega|}{e}\right) .
\end{aligned}
$$

On the other hand,

$$
\frac{\left|\nabla u^{\epsilon}\right|^{2}}{u^{\epsilon}\left(1-\epsilon u^{\epsilon}\right)} \geq \frac{\left|\nabla u^{\epsilon}\right|^{2}}{u^{\epsilon}}
$$

and Corollary 2.3 readily follows from Lemma 2.2 and the previous two inequalities.

Next, for $\epsilon=0$, we may proceed as in [4, Lemma 2.1] to establish a connection between $F_{0}\left(u^{0}, v^{0}\right)$ and the right-hand side of $(5)$.

LEMMA 2.4. If $\epsilon=0$, the condition

$$
\sup _{t \in\left[0, t_{0}^{+}\right)}\left\|u^{0}\right\|_{n / 2} \leq A
$$

for some $A>0$ ensures that the functional $G$ given by

$$
G(u, v)=\int_{\Omega}\left(u \ln \left(\frac{u}{\bar{u}}\right)+\frac{1}{2} \int_{\Omega}|\nabla v|^{2}\right) d x
$$

satisfies the following decay property

$$
0 \leq G\left(u^{0}(t), v^{0}(t)\right) \leq G\left(u_{0}, v_{0}\right) e^{-\alpha t} \quad \text { for } \quad t \in\left[0, t_{0}^{+}\right),
$$

the positive constant $\alpha$ depending only on $\Omega$ and $A$.

Proof. First the non-negativity of $G$ follows from Jensen's inequality as $u^{0}(t)$ and $\bar{u}$ have the same mass $\left\|u_{0}\right\|_{1}$. Next, recalling that

$$
r \ln r-r+1 \leq \frac{(r-1)^{2}}{2} \text { for } r \geq 0,
$$


we infer from the Sobolev, Poincaré and Hölder inequalities that

$$
\begin{aligned}
\int_{\Omega} u^{0}(t) \ln \left(\frac{u^{0}(t)}{\bar{u}}\right) d x & \leq \bar{u} \int_{\Omega}\left[\frac{u^{0}(t)}{\bar{u}}-1+\frac{1}{2}\left(\frac{u^{0}(t)}{\bar{u}}-1\right)^{2}\right] d x \\
& \leq \frac{1}{2 \bar{u}}\left\|u^{0}(t)-\bar{u}\right\|_{2}^{2} \\
& \leq C\left\|\nabla\left(u^{0}(t)-\bar{u}\right)\right\|_{2 n /(n+2)}^{2} \\
& \leq C\left\|\sqrt{u^{0}(t)} \nabla \sqrt{u^{0}(t)}\right\|_{2 n /(n+2)}^{2} \\
& \leq C\left\|u^{0}(t)\right\|_{n / 2}\left\|\nabla \sqrt{u^{0}(t)}\right\|_{2}^{2} \\
& \leq A C \int_{\Omega} \frac{\left|\nabla u^{0}(t)\right|^{2}}{u^{0}(t)} d x .
\end{aligned}
$$

Consequently

$$
G\left(u^{0}(t), v^{0}(t)\right) \leq \frac{1}{\alpha} \int_{\Omega}\left(\frac{\left|\nabla u^{0}(t)\right|^{2}}{u^{0}(t)}+\left|\nabla v^{0}(t)\right|^{2}\right) d x, \quad t \in\left[0, t_{0}^{+}\right) .
$$

We then infer from Lemma 2.2 that

$$
\frac{d}{d t} G\left(u^{0}, v^{0}\right)=\frac{d}{d t} F_{0}\left(u^{0}, v^{0}\right) \leq-\alpha G\left(u^{0}, v^{0}\right),
$$

which completes the proof.

THEOREM 2.5. The only non-negative stationary solutions to (1) in $W^{1, p_{0}}(\Omega)$ for $p_{0}>n$ are the pairs $(m, m)$ for $m \in[0, \infty)$.

Proof. Assume that $\left(u^{0}, v^{0}\right) \in W^{1, p_{0}}\left(\Omega ; \mathbb{R}^{2}\right)$ for $p_{0}>n$ is a stationary solution to (1). Then $t_{0}^{+}=+\infty$ by Theorem 2.1 and it follows from Lemma 2.4 that $0 \leq G\left(u^{0}, v^{0}\right) \leq$ $G\left(u^{0}, v^{0}\right) e^{-\alpha t}$ for each $t \geq 0$, whence $G\left(u^{0}, v^{0}\right)=0$. Consequently,

$$
\nabla v^{0}=0 \text { and } u^{0} \ln \left(\frac{u^{0}}{\bar{u}}\right)=0 \text { a.e. in } \Omega
$$

with $\bar{u}=\left\|u^{0}\right\|_{1} /|\Omega|$, from which we readily conclude that $u^{0}=\bar{u}$ and $v^{0}$ is a constant. Taking into account the second equation in (1) implies that $\left(u^{0}, v^{0}\right)=(m, m)$ for some non-negative real number $m$.

3. The two-dimensional case $n=2$. In this section, we assume that $n=2$ and put $(u, v)=\left(u^{0}, v^{0}\right)$ to simplify the notations, $\left(u^{0}, v^{0}\right)$ being the solution to (4) with $\epsilon=0$ on $\left[0, t_{0}^{+}\right)$given by Theorem 2.1 . We recall that, thanks to Theorem 2.1 , it is sufficient to establish $L^{\infty}$-bounds for $(u, v)$. The following lemma is a first step in that direction.

LEMma 3.1. Let $p \geq 2$ and $T>0$. Then there exists a positive constant $C_{1}(p)$ depending only on $\Omega, u_{0}, v_{0}$ and $p$ such that

$$
\|u(t)\|_{p} \leq C_{1}(p) \quad \text { for } t \in\left[0, t_{0}^{+}\right) .
$$

Proof. We first observe that Corollary 2.3 implies that

$$
\int_{0}^{t} \int_{\Omega}|\Delta v|^{2} d x d t \leq C_{0} \quad \text { for } t \in\left[0, t_{0}^{+}\right)
$$


We next multiply the first equation of $(1)$ by $(p+1) u^{p}$, integrate with respect to the space variable and apply the Gagliardo-Nirenberg inequality (2). We thus obtain

$$
\begin{aligned}
\frac{d}{d t}\left\|u^{(p+1) / 2}\right\|_{2}^{2} & =-\frac{4 p}{p+1}\left\|\nabla\left(u^{(p+1) / 2}\right)\right\|_{2}^{2}+p\left\|u^{p+1} \Delta v\right\|_{1} \\
& \leq-2\left\|\nabla\left(u^{(p+1) / 2}\right)\right\|_{2}^{2}+p\left\|u^{(p+1) / 2}\right\|_{4}^{2}\|\Delta v\|_{2} \\
& \leq-2\left\|\nabla\left(u^{(p+1) / 2}\right)\right\|_{2}^{2}+C p\left\|u^{(p+1) / 2}\right\|_{1,2}\left\|u^{(p+1) / 2}\right\|_{2}\|\Delta v\|_{2} \\
& \leq-\left\|\nabla\left(u^{(p+1) / 2}\right)\right\|_{2}^{2}+\left\|u^{(p+1) / 2}\right\|_{2}^{2}+C p^{2}\left\|u^{(p+1) / 2}\right\|_{2}^{2}\|\Delta v\|_{2}^{2} .
\end{aligned}
$$

Owing to (9) we may apply the Gronwall lemma. The bound is independent of time since we can choose $p=1$ (so that $\frac{p+1}{2}=1$ ) and use the Gagliardo-Nirenberg inequality (2) with (3). Then recursively we get the independent bound for any $p<\infty$. The proof of Lemma 3.1 is completed.

Proof of Theorem 1.1. Owing to Lemma 3.1 we may proceed as in [11, Section 4] and use Moser's iteration technique [1] to show that,

$$
\|u(t)\|_{\infty}+\|v(t)\|_{\infty} \leq C \text { for } t \in\left[0, t_{0}^{+}\right)
$$

According to the global existence criterion from Theorem 2.1, we have thus shown that $t_{0}^{+}=+\infty$. In addition,

$$
(u, v) \in C\left(\bar{\Omega} \times[0, \infty) ; \mathbb{R}^{2}\right) \cap C^{\infty}\left(\bar{\Omega} \times(0, \infty) ; \mathbb{R}^{2}\right),
$$

while LaSalle's invariance principle and (3) ensure that $(u(t), v(t))$ converges towards $(\bar{u}, \bar{v})$ as $t \rightarrow \infty$. For the rate of convergence we may apply Lemma 2.4 thanks to (3) and use the Csiszár-Kullback-Pinsker inequality (see, e.g., [3] and the references therein) to obtain

$$
\frac{1}{2 \bar{u}}\|u-\bar{u}\|_{1}^{2} \leq G(u(t), v(t)) \leq G\left(u_{0}, v_{0}\right) e^{-\alpha t}
$$

and hence the exponential convergence in $L^{1}(\Omega)$.

Since $\bar{u}$ is a constant and $\nabla \cdot(u \nabla v)$ is bounded, the exponential convergence in $L^{\infty}$ may next be proved by Moser's iteration technique [1]. Parabolic estimates then yield the exponential convergence in $W^{2, p}(\Omega)$ for $p>n$.

4. Global weak solutions in higher space dimensions. This section is devoted to the proofs of Theorems 1.3 and 1.4. Both are based on a compactness method. Namely, we shall prove that at least a subsequence of the classical solutions $\left(u^{\epsilon}, v^{\epsilon}\right)$ to $(4)$ converges in suitable topologies towards a (weak) solution to (1) as $\epsilon \rightarrow 0$. As a first step we deduce some bounds on $\left(u^{\epsilon}, v^{\epsilon}\right)$ from Corollary 2.3 and Sobolev embeddings. 
LEMma 4.1. Let $T>0$. The sequences $\left(u^{\epsilon}\right)_{\epsilon}$ and $\left(v^{\epsilon}\right)_{\epsilon}$ enjoy the following properties:

$\left(u^{\epsilon}\right)_{\epsilon} \quad$ is bounded in $L^{(n+2) /(n+1)}\left(0, T ; W^{1,(n+2) /(n+1)}(\Omega)\right)$,

$\left(u_{t}^{\epsilon}\right)_{\epsilon} \quad$ is bounded in $L^{1}\left(0, T ; C_{0}^{1}(\Omega)^{\prime}\right)$,

$\left(v^{\epsilon}\right)_{\epsilon} \quad$ is bounded in $L^{(n+2) / n}\left(0, T ; W^{2,(n+2) / n}(\Omega)\right)$,

$\left(v^{\epsilon}\right)_{\epsilon} \quad$ is bounded in $L^{\infty}\left(0, T ; W^{1,2}(\Omega)\right) \cap L^{2}\left(0, T ; W^{2,2}(\Omega)\right)$,

$\left(v_{t}^{\epsilon}\right)_{\epsilon} \quad$ is bounded in $L^{(n+2) / n}(\Omega \times(0, T))$,

$\left(u^{\epsilon} \nabla v^{\epsilon}\right)_{\epsilon} \quad$ is bounded in $\quad L^{(2 n+4) / 3 n}(\Omega \times(0, T))$.

Proof. Consider $\epsilon \in\left(0, \epsilon_{0}\right)$. We first recall that

$$
\left\|u^{\epsilon}(t)\right\|_{1} \leq K \quad \text { for } t \in[0, T]
$$

by (3). Next, since $\nabla \sqrt{u^{\epsilon}}=\nabla u^{\epsilon} /\left(2 \sqrt{u^{\epsilon}}\right)$, we infer from (16) and Corollary 2.3 that

$$
\int_{0}^{T}\left\|\sqrt{u^{\epsilon}}(t)\right\|_{1,2}^{2} d t \leq K .
$$

The continuous embedding of $W^{1,2}(\Omega)$ in $L^{2 n /(n-2)}(\Omega)$ then entails that

$$
\int_{0}^{T}\left\|u^{\epsilon}(t)\right\|_{n /(n-2)} d t \leq K(T) .
$$

Interpolating between (16) and (17) we obtain

$$
\int_{0}^{T}\left\|u^{\epsilon}(t)\right\|_{n p /(n p-2)}^{p} d t \leq K(T, p) \quad \text { for } p \in[1, \infty] .
$$

In particular, the choice $p=(n+2) / n$ gives

$$
\int_{0}^{T} \int_{\Omega}\left(u^{\epsilon}\right)^{(n+2) / n} d x d t \leq K(T) .
$$

Next, by Corollary 2.3, (19) and the Hölder inequality, we have

$$
\begin{aligned}
\int_{0}^{T} \int_{\Omega}\left|\nabla u^{\epsilon}\right|^{(n+2) /(n+1)} & \leq\left(\int_{0}^{T} \int_{\Omega} \frac{\left|\nabla u^{\epsilon}\right|^{2}}{u^{\epsilon}}\right)^{(n+2) /(2 n+2)}\left(\int_{0}^{T} \int_{\Omega}\left(u^{\epsilon}\right)^{(n+2) / n}\right)^{n /(2 n+2)} \\
& \leq K(T) .
\end{aligned}
$$

We have thus established (10). The bounds (12) and (14) then readily follow from the second equation of (4), (19) and classical parabolic regularity results while Corollary 2.3 and (3) ensure that (13) holds true. We then infer from (13) and the Sobolev embedding that $\left(\nabla v^{\epsilon}\right)_{\epsilon}$ is bounded in both $L^{\infty}\left(0, T ; L^{2}(\Omega)\right)$ and $L^{2}\left(0, T ; L^{2 n /(n-2)}(\Omega)\right)$, whence

$$
\int_{0}^{T}\left\|\nabla v^{\epsilon}\right\|_{2 n p /(n p-4)}^{p} d t \leq K(T, p) \quad \text { for } \quad p \in[2, \infty]
$$

by interpolation. Combining this estimate for $p=2(n+2) / n$ with (19) yields (15). 
Consider finally $\phi \in C_{0}^{1}(\Omega)$. It follows from the first equation of (4) and Corollary 2.3 that

$$
\begin{aligned}
\left|\int_{\Omega} u_{t}^{\epsilon} \phi d x\right| & \leq \int_{\Omega}\left|\nabla u^{\epsilon}\right||\nabla \phi| d x+\int_{\Omega} u^{\epsilon}\left(1-\epsilon u^{\epsilon}\right)\left|\nabla v^{\epsilon}\right||\nabla \phi| d x \\
& \leq\left\|\nabla u^{\epsilon}\right\|_{(n+2) /(n+1)}\|\nabla \phi\|_{\infty}+\left\|u^{\epsilon}\right\|_{2}\left\|\nabla v^{\epsilon}\right\|_{2}\|\nabla \phi\|_{\infty} \\
& \leq K(T)\left(\left\|\nabla u^{\epsilon}\right\|_{(n+2) /(n+1)}+\left\|u^{\epsilon}\right\|_{2}\right)\|\nabla \phi\|_{\infty} .
\end{aligned}
$$

Therefore,

$$
\left\|u_{t}^{\epsilon}\right\|_{C_{0}^{1}(\Omega)^{\prime}} \leq K(T)\left(\left\|\nabla u^{\epsilon}\right\|_{(n+2) /(n+1)}+\left\|u^{\epsilon}\right\|_{2}\right),
$$

and the right-hand side of the above inequality is bounded in $L^{1}(0, T)$ by (17) since $n /(n-2) \geq 2$ for $n=3,4$. The proof of Lemma 4.1 is then complete.

We next turn to the relative compactness of the sequences $\left(u^{\epsilon}\right)_{\epsilon}$ and $\left(v^{\epsilon}\right)_{\epsilon}$. More specifically, we have the following result:

\section{LEMMA 4.2. There are non-negative functions}

$$
\begin{aligned}
& u \in L^{(n+2) /(n+1)}\left(0, T ; W^{1,(n+2) /(n+1)}(\Omega)\right) \cap C\left([0, T] ; C_{0}^{1}(\Omega)^{\prime}\right), \quad u(0)=u_{0}, \\
& v \in L^{\infty}\left(0, T ; H^{1}(\Omega)\right) \cap C\left([0, T] ; L^{2}(\Omega)\right) \cap L^{2}\left(0, T ; W^{2,2}(\Omega)\right), \quad v(0)=v_{0},
\end{aligned}
$$

and a subsequence of $\left(u^{\epsilon}\right)_{\epsilon}$ and $\left(v^{\epsilon}\right)_{\epsilon}$ (not relabeled) such that

$$
\begin{aligned}
& u^{\epsilon} \rightarrow u \quad \text { in } \quad L^{p}(\Omega \times(0, T)) \cap C\left([0, T] ; C_{0}^{1}(\Omega)^{\prime}\right) \quad \text { for } p \in\left[1, \frac{n+2}{n}\right), \\
& v^{\epsilon} \rightarrow v \quad \text { in } \quad L^{2}\left(0, T ; H^{1}(\Omega)\right) \cap C\left([0, T] ; L^{2}(\Omega)\right),
\end{aligned}
$$

and

$$
\int_{\Omega}\left(v(t)-v_{0}\right) \varphi d x+\int_{0}^{t} \int_{\Omega}(\nabla v \cdot \nabla \varphi+(v-u) \varphi) d x d s=0
$$

for each $t \in[0, T]$ and $\varphi \in W^{1, \infty}(\Omega)$.

Proof. In view of (10) and (11), we see that $\left(u^{\epsilon}\right)_{\epsilon}$ is relatively compact in $L^{(n+2) /(n+1)}(\Omega \times$ $(0, T))$ by the Aubin-Lions lemma [8, Théorème 5.1]. In fact we can strengthen this claim due to (19) and deduce that

$$
\left(u^{\epsilon}\right)_{\epsilon} \text { is relatively compact in } L^{p}(\Omega \times(0, T)) \text { for any } p \in\left[1, \frac{n+2}{n}\right) \text {. }
$$

Similarly, it follows from (13), (14), and [13, Corollary 4] that

$$
\left(v^{\epsilon}\right)_{\epsilon} \text { is relatively compact in } C\left([0, T] ; L^{2}(\Omega)\right) \cap L^{2}\left(0, T ; W^{1,2}(\Omega)\right) .
$$

Owing to (21) and (22) we easily obtain the convergences claimed in Lemma 4.2, the convergence of $\left(u^{\epsilon}\right)_{\epsilon}$ in $C\left([0, T] ; C_{0}^{1}(\Omega)^{\prime}\right)$ being a consequence of (11), (16), and the Ascoli theorem. It is then straightforward to pass to the limit as $\epsilon \rightarrow 0$ in the second equation of (4) to deduce the last assertion of Lemma 4.2.

It remains to pass to the limit as $\epsilon \rightarrow 0$ in the first equation of (4), the main difficulty being the nonlinear term $u^{\epsilon}\left(1-\epsilon u^{\epsilon}\right) \nabla v^{\epsilon}$. At this point the difference between $n=3$ and $n=4$ shows up: indeed, though we know that

$$
u^{\epsilon}\left(1-\epsilon u^{\epsilon}\right) \nabla v^{\epsilon} \rightarrow u \nabla v \quad \text { a.e. in } \Omega \times(0, T)
$$


by Lemma 4.2 (after possibly extracting a further subsequence), we only have an $L^{1}$ bound for this term when $n=4$ by (15) and this is not sufficient to have strong convergence. Such a difficulty is not encountered when $n=3$ and we now complete the proof of Theorem 1.3.

Proof of Theorem 1.3. According to (15), the sequence $\left(u^{\epsilon}\left(1-\epsilon u^{\epsilon}\right) \nabla v^{\epsilon}\right)_{\epsilon}$ is bounded in $L^{10 / 9}(\Omega \times(0, T))$ and thus weakly compact in $L^{1}(\Omega \times(0, T))$. Since it also converges a.e. in $\Omega \times(0, T)$ by $(23)$, we are in a position to apply the Vitali theorem and conclude that

$$
u^{\epsilon}\left(1-\epsilon u^{\epsilon}\right) \nabla v^{\epsilon} \rightarrow u \nabla v \quad \text { in } \quad L^{1}(\Omega \times(0, T)) .
$$

In view of Lemma 4.2 it is then straightforward to let $\epsilon \rightarrow 0$ in the first equation of (4) and conclude that $(u, v)$ is a weak solution to (1) in the sense of Definition 1.2.

We next turn to the convergence towards steady states. We first recall that the $L^{1}$ norms of $(u, v)$ are given by (3). It follows from Lemma 4.2 and weak compactness arguments that we may pass to the limit as $\epsilon \rightarrow 0$ in the inequality stated in Corollary 2.3 to obtain that

$$
\int_{\Omega}\left(u(t)|\ln u(t)|+\frac{|\nabla v(t)|^{2}}{2}\right) d x+\int_{0}^{t} \int_{\Omega}\left(4|\nabla \sqrt{u}|^{2}+|\Delta v|^{2}+|\nabla v|^{2}\right) d x d s \leq C_{0} .
$$

Next, we take $0=t_{0} \leq t_{1} \leq \cdots \leq t_{k}$ with $t_{k} \rightarrow+\infty$ and we define

$$
\begin{gathered}
u_{k}(\cdot, t)=u\left(\cdot, t+t_{k}\right)-\left\langle u_{0}\right\rangle, \quad t \in(0,1) \\
v_{k}(\cdot, t)=v\left(\cdot, t+t_{k}\right)-\left\langle v\left(t+t_{k}\right)\right\rangle \quad t \in(0,1)
\end{gathered}
$$

where $\langle\cdot\rangle$ denotes the mean value. We infer from the Dunford-Pettis theorem and (24) that

$$
\left(u_{k}(0), v_{k}(0)\right) \rightarrow\left(u_{\infty}, v_{\infty}\right) \text { weak- } L^{1}(\Omega) \times L^{2}(\Omega) .
$$

Then, taking into account that

$$
\left(\int_{\Omega}|\nabla u| d x\right)^{2} \leq C \int_{\Omega} \frac{|\nabla u|^{2}}{u} d x
$$

and (24) we deduce

$$
\left(\nabla u_{k}, \nabla v_{k}\right) \rightarrow(0,0) \text { in } L^{2}\left(0,1 ; L^{1}(\Omega)\right) \times L^{2}\left(0,1 ; H^{1}(\Omega)\right) .
$$

Hence by the Poincaré-Wirtinger inequality

$$
\left(u_{k}, v_{k}\right) \rightarrow(0,0) \text { in } L^{1}\left(0,1 ; L^{1}(\Omega)\right) .
$$

On the other hand, using the definition of a weak solution, the embeddings $W^{1,1}(\Omega)$ in $L^{4 / 3}(\Omega), W^{1,2}(\Omega)$ in $L^{2}(\Omega)$ and $(26)$ we prove

$$
\begin{aligned}
& \lim _{k \rightarrow+\infty} \sup _{t \in[0,1]}\left|\int_{\Omega}\left(u_{k}(t)-u_{k}(0)\right) \varphi d x\right|=0 \quad \forall \varphi \in W^{1, \infty}(\Omega), \\
& \lim _{k \rightarrow+\infty} \sup _{t \in[0,1]}\left|\int_{\Omega}\left(v_{k}(t)-v_{k}(0)\right) \varphi d x\right|=0 \quad \forall \varphi \in W^{1, \infty}(\Omega) .
\end{aligned}
$$

In view of (27), (28) and (29) we can identify the limits in (25) as being zero. 
Proof of Theorem 1.4. In that case the weak compactness in $L^{1}(\Omega \times(0, T))$ of $\left(u^{\epsilon}\left(1-\epsilon u^{\epsilon}\right) \nabla v^{\epsilon}\right)_{\epsilon}$ is no longer guaranteed by (15) and we thus have to find an alternative way to prove it. To this end, we aim at applying the Dunford-Pettis theorem and first notice that $\left(u^{\epsilon}\right)_{\epsilon}$ actually enjoys a stronger property than (3), namely

$$
\sup _{t \in[0, T]}\left\{\int_{\Omega} u^{\epsilon}(t)\left|\ln u^{\epsilon}(t)\right| d x\right\} \leq C_{0}
$$

by Corollary 2.3. Thanks to this property, we can establish the uniform integrability of $\left(u^{\epsilon}\left(1-\epsilon u^{\epsilon}\right) \nabla v^{\epsilon}\right)_{\epsilon}$. Indeed, let $E \subset \Omega \times(0, T)$ and $R>1$. We infer from (18) with $p=2$, (20) with $p=2$, and (30) that

$$
\begin{aligned}
& \iint_{E} u^{\epsilon}\left(1-\epsilon u^{\epsilon}\right) \nabla v^{\epsilon} d x d t \leq \iint_{E} u^{\epsilon} \nabla v^{\epsilon} d x d t \\
& \leq R \iint_{E} \nabla v^{\epsilon} d x d t+\iint_{E} u^{\epsilon} \mathbf{1}_{(R, \infty)}\left(u^{\epsilon}\right) \nabla v^{\epsilon} d x d t \\
& \leq C R|E|^{1 / 2}+\int_{0}^{T}\left\|u^{\epsilon} \mathbf{1}_{(R, \infty)}\left(u^{\epsilon}\right)\right\|_{4 / 3}\left\|\nabla v^{\epsilon}\right\|_{4} d t \\
& \leq C R|E|^{1 / 2}+\sup _{t \in[0, T]}\left\{\left\|u^{\epsilon}(t) \mathbf{1}_{(R, \infty)}\left(u^{\epsilon}(t)\right)\right\|_{1}\right\}\left\|u^{\epsilon}\right\|_{L^{2}\left(0, T ; L^{4 / 3}(\Omega)\right)}\left\|\nabla v^{\epsilon}\right\|_{L^{2}\left(0, T ; L^{4}(\Omega)\right)} \\
& \leq C R|E|^{1 / 2}+\frac{C}{\ln R} \sup _{t \in[0, T]}\left\{\left\|u^{\epsilon}(t)\left|\ln u^{\epsilon}(t)\right|\right\|_{1}\right\} \\
& \leq C R|E|^{1 / 2}+\frac{C}{\ln R} .
\end{aligned}
$$

Letting first $|E| \rightarrow 0$ and then $R \rightarrow \infty$ we end up with

$$
\lim _{|E| \rightarrow 0} \sup _{\epsilon \in\left(0, \epsilon_{0}\right)}\left\{\iint_{E} u^{\epsilon}\left(1-\epsilon u^{\epsilon}\right) \nabla v^{\epsilon} d x d t\right\}=0,
$$

which ensures the weak compactness of $\left(u^{\epsilon}\left(1-\epsilon u^{\epsilon}\right) \nabla v^{\epsilon}\right)_{\epsilon}$ in $L^{1}(\Omega \times(0, T))$ by the Dunford-Pettis theorem. Recalling (23) we may apply again the Vitali theorem to conclude that

$$
u^{\epsilon}\left(1-\epsilon u^{\epsilon}\right) \nabla v^{\epsilon} \rightarrow u \nabla v \quad \text { in } \quad L^{1}(\Omega \times(0, T)) .
$$

We then argue as in the proof of Theorem 1.3 to show that $(u, v)$ is a weak solution to $(1)$ in the sense of Definition 1.2 and that $(u(t), v(t))$ converges towards $(\bar{u}, \bar{v})$ in the expected topologies.

Acknowledgments. C. Morales-Rodrigo gratefully acknowledges support from the EU Marie Curie Research Training Network Grant "Modelling, Mathematical Methods and Computer Simulations of Tumour Growth and Therapy", contract number MCRTN-CT2004-503661. Partial support from the French-Polish POLONIUM project 11605VC is also gratefully acknowledged by $\mathrm{T}$. Cieślak and $\mathrm{Ph}$. Laurençot.

\section{References}

[1] N. Alikakos, An application of the invariance principle to reaction-diffusion equations, J. Differential Equations 33 (1979), 203-225. 
[2] H. Amann, Nonhomogeneous linear and quasilinear elliptic and parabolic boundary value problems, in: Function Spaces, Differential Operators and Nonlinear Analysis, H. Triebel and H. J. Schmeisser (eds.), Teubner-Texte Math. 133, Teubner, Stuttgart, 1993, 9-126.

[3] J. A. Carrillo, A. Jüngel, P. Markowich, G. Toscani and A. Unterreiter, Entropy dissipation methods for degenerate parabolic problems and generalized Sobolev inequalities, Monatsh. Math. 133 (2001), 1-82.

[4] H. Gajewski and K. Zacharias, Global behaviour of a reaction-diffusion system modelling chemotaxis, Math. Nachr. 195 (1998), 77-114.

[5] D. Horstmann, From 1970 until present: the Keller-Segel model in chemotaxis and its consequences. I, Jahresber. Deutsch. Math.-Verein. 105 (2003), 103-165.

[6] W. Jäger and S. Luckhaus, On explosions of solutions to a system of partial differential equations modelling chemotaxis, Trans. Amer. Math. Soc. 329 (1992), 819-824.

[7] E. F. Keller and L. A. Segel, Initiation of slime mold aggregation viewed as an instability, J. Theor. Biology 26 (1970), 399-415.

[8] J. L. Lions, Quelques Méthodes de Résolution des Problèmes aux Limites non Linéaires, Dunod, Paris, 1969.

[9] M. S. Mock, An initial value problem from semiconductor device theory, SIAM J. Math. Anal. 5 (1974), 597-612.

[10] M. S. Mock, Asymptotic behavior of solutions of transport equations for semiconductor devices, J. Math. Anal. Appl. 49 (1975), 215-225.

[11] T. Nagai, T. Senba and K. Yoshida, Application of the Trudinger-Moser inequality to a parabolic system of chemotaxis, Funkcial. Ekvac. 40 (1997), 411-433.

[12] J. Rencławowicz and T. Hillen, Analysis of an attraction-repulsion chemotaxis model, preprint.

[13] J. Simon, Compact sets in the space $L^{p}(0, T ; B)$, Ann. Mat. Pura Appl. 146 (1987), 65-96. 
